\title{
Erratum: Cryptographic quantum bound on nonlocality [Phys. Rev. A 95, 022108 (2017)]
}

\author{
Satoshi Ishizaka
}

(Received 22 February 2018; published 8 March 2018)

DOI: 10.1103/PhysRevA.97.039902

For quantum states $(0 \leqslant \lambda \leqslant 1)$,

$$
\rho=\lambda \rho_{0} \otimes|0\rangle\left\langle 0\left|+(1-\lambda) \rho_{1} \otimes\right| 1\right\rangle\left\langle 1\left|, \quad \sigma=\lambda \sigma_{0} \otimes\right| 0\right\rangle\left\langle 0\left|+(1-\lambda) \sigma_{1} \otimes\right| 1\right\rangle\langle 1|,
$$

the generalized trace distancelike quantity $\tilde{D}$, defined as

$$
\tilde{D}(\rho, \sigma)=\max _{X} \frac{\operatorname{tr} X(\rho-\sigma)}{\sqrt{\operatorname{tr} X^{2}(\rho+\sigma)}}
$$

behaves as $\tilde{D}^{2}(\rho, \sigma)=\lambda \tilde{D}^{2}\left(\rho_{0}, \sigma_{0}\right)+(1-\lambda) \tilde{D}^{2}\left(\rho_{1}, \sigma_{1}\right)$, contrary to $\bar{D}(\rho, \sigma) \equiv \operatorname{tr}|\rho-\sigma|=\lambda \bar{D}\left(\rho_{0}, \sigma_{0}\right)+(1-\lambda) \bar{D}\left(\rho_{1}, \sigma_{1}\right)$. As a result, for a quantum correlation of the form $\mathbf{q}=\lambda \mathbf{p}+(1-\lambda) \mathbf{I}$, there always exists a realization with $\tilde{D}^{\mathbf{q}}=\sqrt{\lambda} \tilde{D}_{x}^{\mathbf{p}}$ and $\bar{D}^{\mathbf{q}}=\lambda \bar{D}_{x}^{\mathbf{p}}$, and $\tilde{C}_{x y}$ is invariant only when $C_{x y}$ is scaled by $\bar{D}_{x}$. Therefore, $\tilde{D}_{x}$ should be read as $\bar{D}_{x}$ in Lemma 2 and Fig. 2 where the saturation of Eq. (6) inside the set of quantum correlations is discussed. Such a replacement is justified only when Eq. (6) is known to be saturated at extremal points of the set of quantum correlations, where $\tilde{D}_{x}=\bar{D}_{x}$. This is indeed the case for Lemma 2 and Fig. 2. 